\title{
Review Article \\ Kinesio Taping for Balance Function after Stroke: A Systematic Review and Meta-Analysis
}

\author{
Yijuan Hu, ${ }^{1}$ Dongling Zhong $\mathbb{D}^{1},{ }^{1}$ Qiwei Xiao, ${ }^{1}$ Qiang Chen, \\ Juan Li $\odot{ }^{1}{ }^{1}$ and Rongjiang Jin $\oplus^{1}$ \\ ${ }^{1}$ Chengdu University of Traditional Chinese Medicine, Chengdu, Sichuan Province, China \\ ${ }^{2}$ Huili County People's Hospital, Sichuan Province, China \\ Correspondence should be addressed to Juan Li; 785939016@qq.com and Rongjiang Jin; jrj133@126.com
}

Received 16 January 2019; Revised 10 June 2019; Accepted 2 July 2019; Published 16 July 2019

Academic Editor: Jenny M. Wilkinson

Copyright (C) 2019 Yijuan Hu et al. This is an open access article distributed under the Creative Commons Attribution License, which permits unrestricted use, distribution, and reproduction in any medium, provided the original work is properly cited.

Objective. With the increasing social and economic burdens of balance impairment after stroke, the treatment for balance impairment after stroke becomes a major public health problem worldwide. Kinesio taping (KT) as a part of clinical practice has been used widely in the treatment of balance impairment after stroke. However, the clinical effects of KT for balance function have not been confirmed. The objective of this study is to investigate the effects and safety of KT for balance impairment after stroke. Methods. We conducted a systematic review (SR) and meta-analysis of randomized controlled trials (RCTs) on the effects of KT for balance impairment after stroke. We searched the following databases: (1) English databases: EMBASE (via Ovid), MEDLINE (via Ovid), the Cochrane library, PubMed, and PEDro; (2) Chinese databases: China Biology Medicine (CBM), Wan Fang database, China National Knowledge Infrastructure (CNKI), and VIP. Besides, hand searches of relevant references were also conducted. We systematically searched from the inception to December 2018, using the keywords (Kinesio, Kinesio Tape, tape, or Orthotic Tape) and (stroke, hemiplegia, or hemiplegic paralysis) and (balance or stability). The search strategies were adjusted for each database. The reference lists of included articles were reviewed for relevant trials. For missing data, we contacted the authors to get additional information. Results. 22 RCTs involved 1331 patients, among which 667 patients in the experimental group and 664 patients in the control group were included. Results of meta-analysis showed that, compared with conventional rehabilitation (CR), there was significant difference in Berg Balance Scale (BBS) ( $\mathrm{MD}=4.46$, 95\%CI 1.72 to 7.19, $P=0.001$ ), Time Up and Go Test (TUGT) (MD=$4.62,95 \% \mathrm{CI}-5.48$ to $-3.79, P<0.00001$ ), functional ambulation category scale (FAC) (MD=0.53, $95 \% \mathrm{CI} 0.38$ to $0.68, P<0.00001$ ), Fugl-Meyer assessment (FMA-L) $(\mathrm{MD}=4.20,95 \% \mathrm{CI} 3.17$ to 5.24, $P<0.00001)$, and Modified Ashworth Scale (MAS) (MD=-0.38, $95 \% \mathrm{CI}-0.49$ to $-0.27, P<0.00001)$. The results of subgroup analysis showed that there was no significant difference between KT and $\mathrm{CR}$ with $\leq 4$ weeks treatment duration ( $<4$ weeks: $\mathrm{MD}=5.03,95 \% \mathrm{CI}-1.80$ to $11.85, P=0.15 ;=4$ weeks: $\mathrm{MD}=4.33,95 \% \mathrm{CI}-1.50$ to 10.15 , $P=0.15$ ), while there was significant difference with more than 4 -week treatment duration (MD $=4.77,95 \% \mathrm{CI} 2.58$ to $6.97, P<0.0001$ ). Conclusions. Based on current evidence, KT was more effective than CR for balance function, lower limb function, and walking function in poststroke patients. Longer treatment duration may be associated with better effects. However, more well-conducted $\mathrm{RCTs}$ are required in the future.

\section{Introduction}

Stroke is a common clinical cerebrovascular disease, including cerebral infarction, intracerebral hemorrhage (ICH), and subarachnoid hemorrhage (SAH), with a high morbidity, which is a major cause of disability and death among people around the world [1]. As the world's population ages, the burden of stroke increases rapidly [2]. WHO Global status report on noncommunicable disease (NCD) showed an estimated 6.7 million NCD deaths were caused by stroke; 33 million of stroke survivors needed long-term follow-up and secondary preventive measures [3]. A study on 84,184 acute stroke patients in England, Wales, and Northern Ireland showed that the total cost of health and social care each year was $\mathfrak{£ 3 . 6 0}$ billion in the first five years after admission [4]. A study on economic burden of informal care attributable to 
stroke among those aged 65 years or older in China showed that the average annual cost of informal care associated with stroke was 10,612 RMB per stroke survivor [5].

Balance impairment is one of the common daily functional problems in stroke patients, which seriously affects the patient's daily life and work. According to the severity of stroke, the manifestations of balance impairment can be classified into stepping, standing, and sitting balance impairment [6]. Poor balance ability is often associated with increased risk of falls, disability, and even mortality. A study based on 41 community-dwelling people with stroke showed $50 \%$ (21/41) participants were classed as fallers, of whom 10 had fallen repeatedly [7]. Another study based on 522 adults showed that, by 2 years after stroke, 23.5\% (124/522) adults had fallen at least once, $14.2 \%$ (74/522) had 2 or more falls and 5.4\% (28/522) had a fracture [8]. Especially for old people, owing to poor balance ability, they have to face the risk of body injuries caused by falls for a long time [9], some severe falls can even lead to paralysis or death. This damage is undoubtedly serious and complicated. Hence, it is urgent for stroke patients to improve their balance ability [1015].

Kinesio taping (KT) was introduced by Kenzo Kase in 1996, which normally involves a combination of applying appropriate tension along the elastic therapeutic tape and placing the target muscle in a stretched position and is widely used as an interesting and relatively novel method for various clinical treatments. KT has gained popularity in clinical practice and been used in clinical practice worldwide. By supporting weak muscle, relaxing overstretched muscle, and reducing pain, KT can promote functional use of the upper and lower extremity, in further to improve balance ability [16]. Besides, KT was also a good option for stroke patients who had asymmetrical and imbalanced body posture [17]. Recent studies showed that KT has been used as an adjuvant rehabilitation therapy in the treatment of balance impairment after stroke [18-36], but the conclusions were inconsistent. The effect of KT on balance impairment of poststroke patients is still controversial. Therefore, a systematic review (SR) and meta-analysis of randomized controlled trials (RCTs) was performed to investigate the efficacy and safety of KT on balance impairment after stroke.

\section{Materials and Methods}

2.1. Data Sources and Searches. We searched the following databases:

(1) English databases: EMBASE (via Ovid), MEDLINE (via Ovid), the Cochrane library, PubMed, and PEDro;

(2) Chinese databases: China Biology Medicine (CBM), Wan Fang database, China National Knowledge Infrastructure (CNKI), and VIP.

Hand searches of relevant references were conducted. Clinical trials registration websites, including https://clinicaltrials.gov/ and http://www.chictr.org.cn, were also searched for possible RCTs.

All the databases were conducted from their inception to December 2018. The search terms (Kinesio, Kinesio Tape, tape, or Orthotic Tape) and (stroke, hemiplegia, or hemiplegic paralysis) and (balance or stability) were used and the search strategies were adjusted for each database. Details of search strategies were given in Appendix A.

2.2. Inclusion Criteria. Trials were screened based on the following inclusion criteria: (1) RCTs of KT for stroke patients with balance impairment; (2) patients with balance impairment after stroke diagnosed according to stroke diagnostic criteria with clear history and manifestations and confirmed by the brain Computed Tomography (CT) or Magnetic Resonance Imaging (MRI) and the age and sex are not limited; (3) the experimental group was treated with KT along with or without conventional rehabilitation (CR). The control group can be CR, sham KT, or placebo; (4) primary outcome is Berg Balance Scale (BBS). Secondary outcomes include related outcomes to lower limb function, spasticity, walking function.

2.3. Exclusion Criteria. Trials met the following criteria would be excluded: (1) Trials were non-RCTs, such as literature review, case report, and expert treatment experience summary; (2) balance impairment was not caused by stroke, for example, caused by Parkinson's disease, pediatric cerebral palsy, knee surgery, or other diseases; (3) duplicate or the data cannot be extracted.

2.4. Studies Selection. All the retrieved studies were imported into Endnote (X8), and the filter tool was used to delete the duplicated studies. Two reviewers (YJH and DLZ) independently screened the titles and abstracts according to the inclusion and exclusion criteria. After screening, two reviewers (YJH and DLZ) cross checked and downloaded the full text of all possibly relevant studies for further assessment. The disagreements were resolved through team discussion.

2.5. Data Extraction. A standardized data extraction form was designed in advance. The following information of the included studies, first author; publication year; country of origin; participant characteristics; evaluation time and outcomes, were independently extracted by two reviewers (QWX and YJH). If the trials had more than 2 groups or factorial designs and multiple comparisons, we extracted only the information and data of interest reported in the original articles. In case of disagreements, a third reviewer (RJJ) was involved.

2.6. Risk-of-Bias Assessments. The methodological quality for the included RCTs was independently assessed by 2 reviewers (YJH and QC) based on Cochrane risk-of-bias criteria [19]. The Cochrane risk-of-bias criteria included the randomization sequence generation, allocation concealment, blinding of participants and personnel, blinding of outcome assessment, incomplete outcome data, selective reporting, and other bias. Each quality item was graded as low, high, or unclear. The disagreements of assessment were resolved through team discussion. 


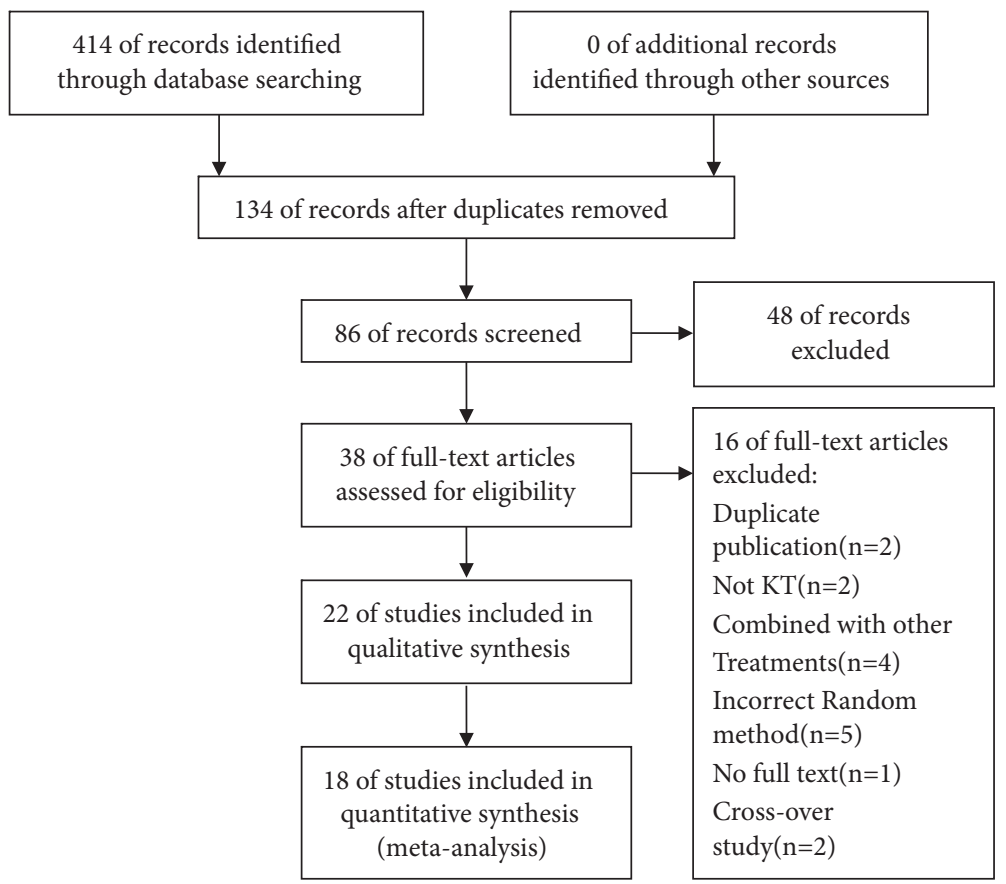

FIGURE 1: PRISMA flow chart for study selection.

2.7. Statistical Analysis. We performed meta-analysis to calculate mean difference (MD) and 95\% CIs using the MantelHaenszel statistical method. Heterogeneity test of each outcome was conducted by using the Chi-square test with no significance $(P>0.05)$, and $I^{2}$ statistic evaluated the degree of heterogeneity. If $I^{2}<50 \%$, many similar studies could be considered to have no homogeneity, the fixed effect model adopted for a meta-analysis; otherwise random effects model was performed. If substantial heterogeneity was detected, subgroup analysis or sensitivity analysis could be applied to explore the causes of heterogeneity. If the sources of heterogeneity could not be determined, descriptive analysis was adopted.

\section{Results}

3.1. Study Selection. A total of 414 potentially relevant articles were retrieved. After removing duplicates, titles and abstracts of remaining articles were screened for inclusion. After reading full texts, 22 RCTs were included in SR and 18 RCTs for meta-analysis. Excluded articles with explanations were listed in Appendix B. Flow diagram for selection of the included studies was showed in Figure 1.

3.2. Studies Description. 22 RCTs involved 1331 patients, among which 667 patients are in the experimental group and 664 patients are in the control group. Included RCTs were published from 2014 to 2019, among which 18 RCTs were conducted in China, 3 were in Korean [18-20], and 1 was in Iran [21]. Disease duration varied from days to years. The control group in 20 RCTs utilized CR, while 2 RCTs used sham KT $[20,22]$. The locations of KT were on lower limbs (quadriceps, triceps surae, tibialis anterior, ankle, and so on). The treatment duration of KT varied from immediate effect to 3 months. Only 1 RCT mentioned follow-up [22]; only 7 RCTs reported funding and sources [21, 25, 28, 32, 35-37]. The characteristics of the included RCTs in detail were showed in Table 1.

3.3. Methodological Quality. The risk of bias assessed by the Cochrane Collaboration's tool of the included RCTs was summarized in Figures 2(a) and 2(b). Among the 22 included RCTs, the randomization procedure was reported adequately in the 8 RCTs; 2 RCTs clearly reported the allocation concealment. Only 1 RCT described the blinding of participants and personnel. We considered RCTs using objective outcome indicator as "low quality"; there were 6 RCTs considered "low quality" in the blinding of outcome assessment. In incomplete outcome data section, only 1 RCT was considered "high quality" due to no reason for loss. All the 22 RCTs were rated "uncertain risks" in selective reporting for no protocol published in advance. In other bias section, we considered "uncertain risks" if the study did not report conflict of interest or source of funds.

\subsection{Meta-Analysis}

\subsubsection{Primary Outcome}

BBS. 8 RCTs compared KT with CR $[18,19,21,22,26,28$, 30,33]. As shown in Figure 3, there was significant difference between $\mathrm{KT}$ and CR in BBS (MD=4.46, 95\%CI 1.72 to 7.19, $P=0.001) ; 2$ RCTs compared KT with sham KT, there was no significant difference $(\mathrm{MD}=3.13,95 \% \mathrm{CI}-0.96$ to $7.23, P=0.13)$. 


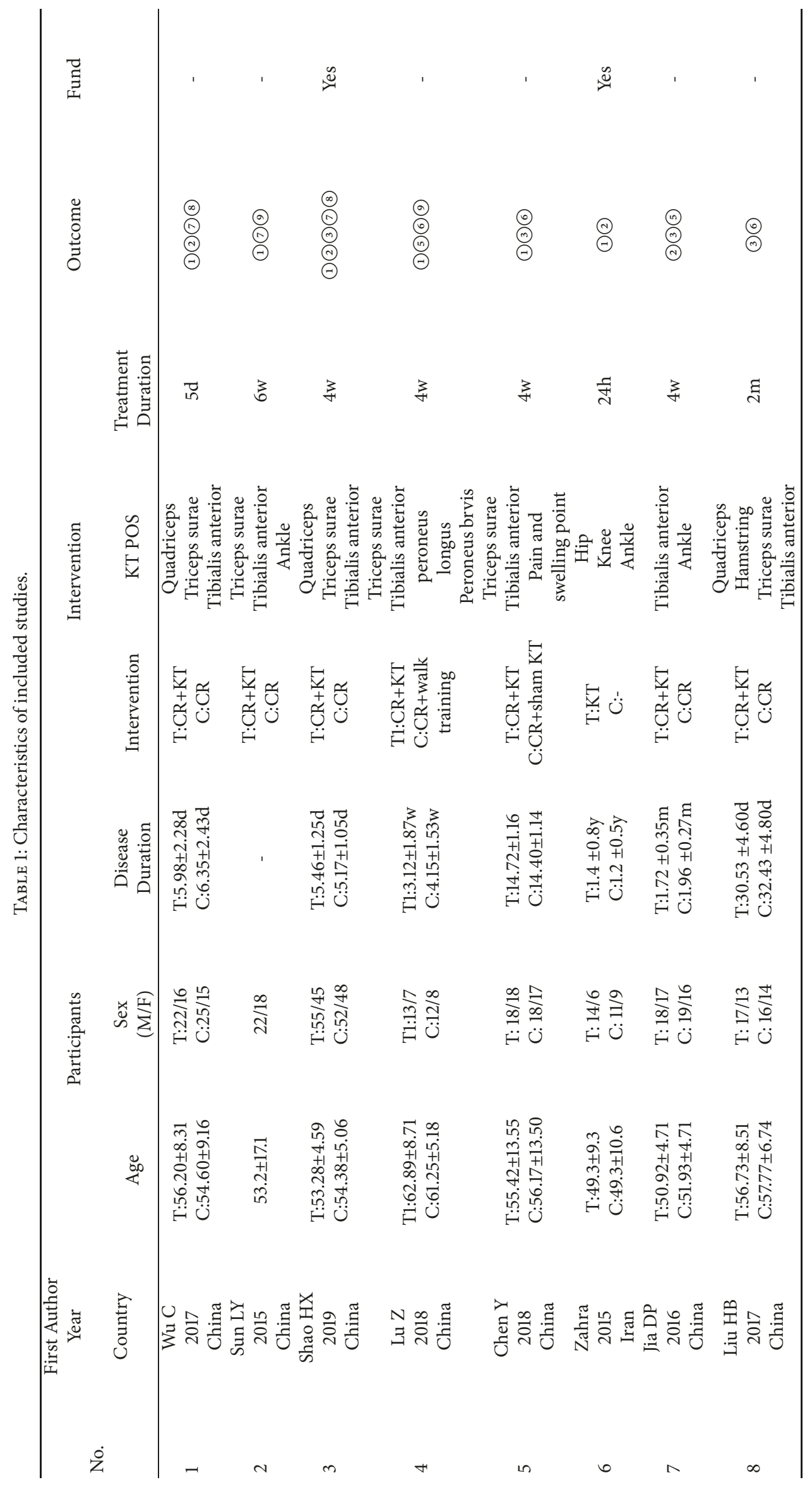




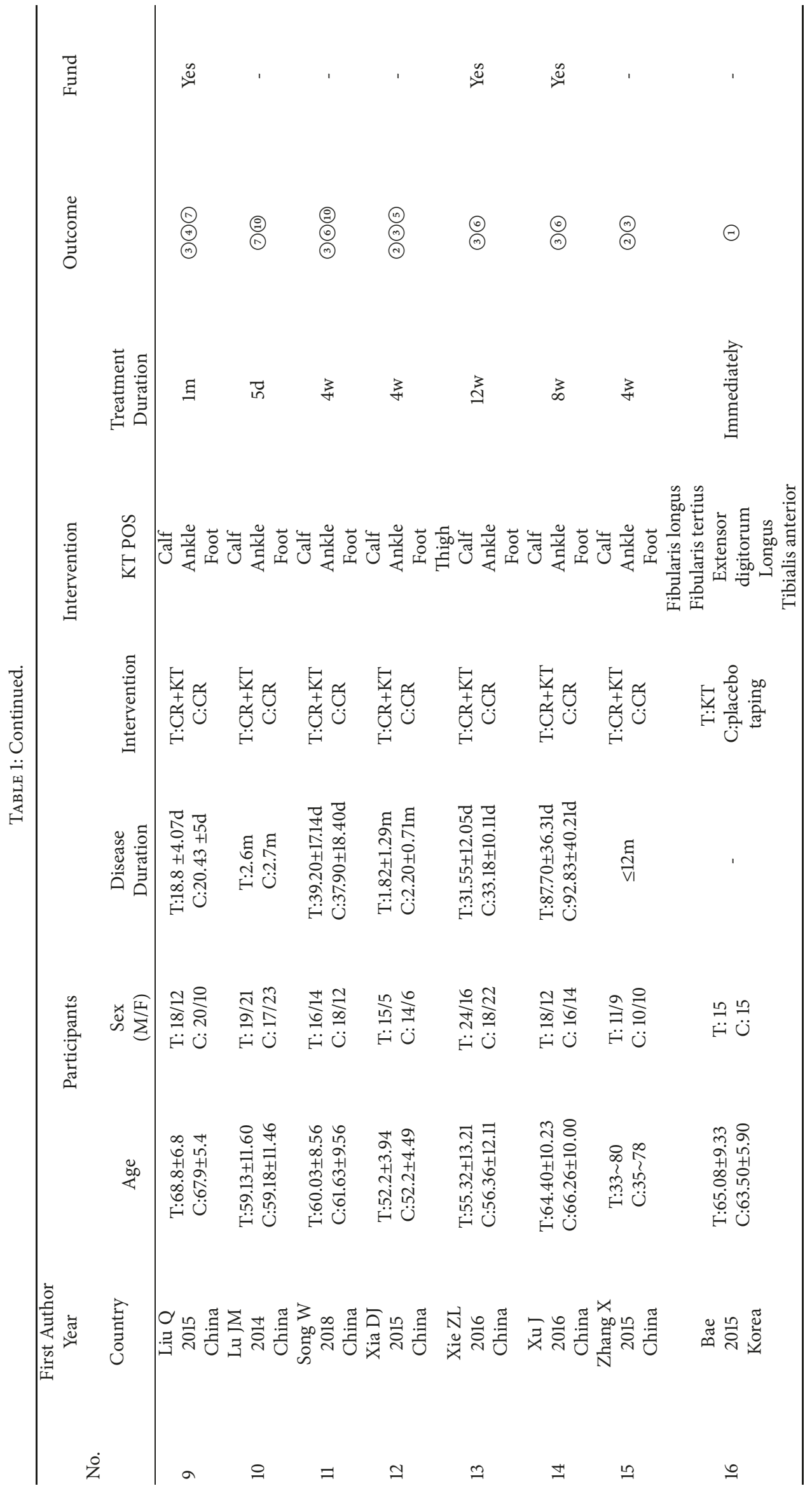




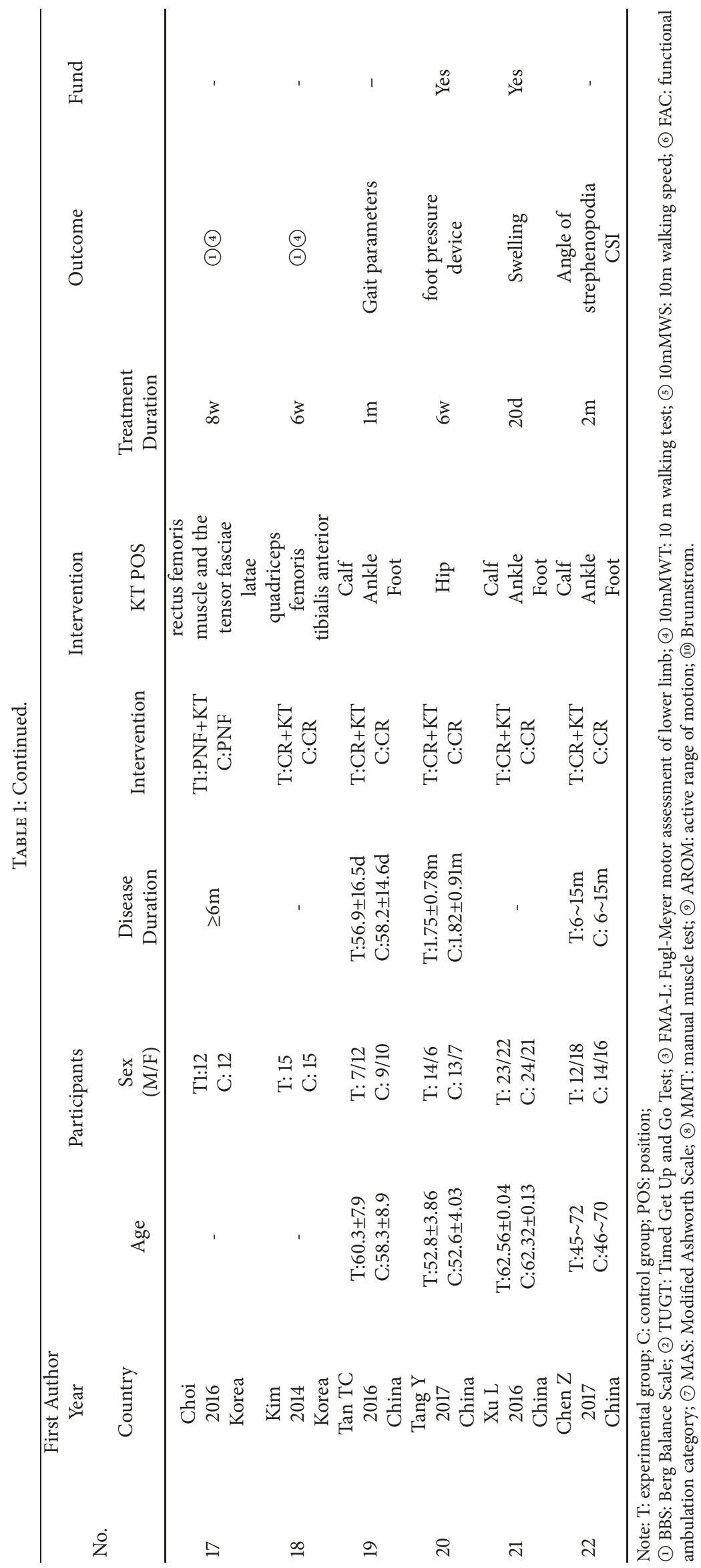




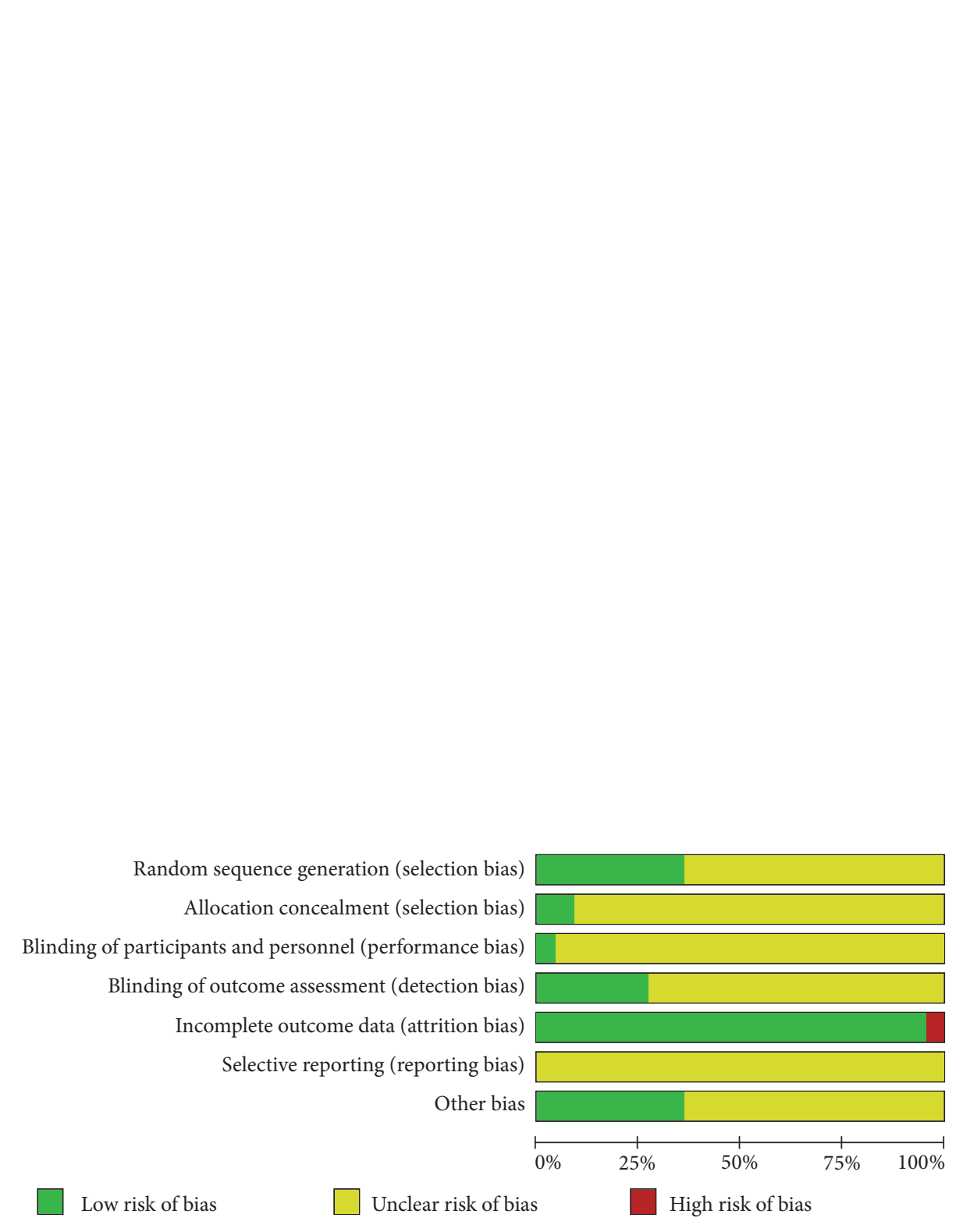

(a)

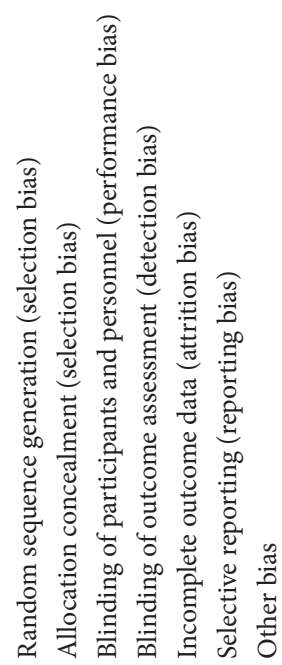

\begin{tabular}{|c|c|c|c|c|c|c|c|}
\hline Bae 2015 & $?$ & $?$ & $\oplus$ & $?$ & + & $?$ & $\oplus$ \\
\hline Chen Y 2018 & + & + & $?$ & + & $\oplus$ & $?$ & $?$ \\
\hline Chen Z 2017 & ? & ? & $?$ & $?$ & $\oplus$ & $?$ & $?$ \\
\hline Choi 2016 & ? & ? & $?$ & ? & $\oplus$ & $?$ & ? \\
\hline Jia DP 2016 & $?$ & $\oplus$ & $?$ & $?$ & 4 & $?$ & $?$ \\
\hline Kim 2014 & $?$ & $?$ & $?$ & $?$ & + & $?$ & $?$ \\
\hline Liu HB 2017 & $?$ & $?$ & $?$ & $?$ & $\oplus$ & $?$ & $\oplus$ \\
\hline Liu Q 2015 & $\oplus$ & $?$ & $?$ & $\oplus$ & $\oplus$ & $?$ & $\oplus$ \\
\hline Lu JM 2014 & + & $?$ & $?$ & $?$ & $\oplus$ & $?$ & $?$ \\
\hline Lu Z 2018 & $\oplus$ & $?$ & $?$ & $\oplus$ & $\oplus$ & $?$ & $?$ \\
\hline hao HX 2019 & $\oplus$ & $?$ & $?$ & $?$ & $\oplus$ & $?$ & $\oplus$ \\
\hline Song W 2018 & $?$ & $?$ & $?$ & $?$ & $\oplus$ & $?$ & $?$ \\
\hline Sun LY 2015 & $?$ & $?$ & $?$ & $?$ & $\oplus$ & $?$ & $?$ \\
\hline Tang Y 2017 & + & $?$ & $?$ & $\oplus$ & $\oplus$ & $?$ & + \\
\hline Tan TC 2016 & $?$ & $?$ & ? & $\oplus$ & $\oplus$ & $?$ & $?$ \\
\hline Wu C 2017 & $?$ & $?$ & $?$ & $?$ & $\theta$ & $?$ & $?$ \\
\hline Xia DJ 2015 & + & $?$ & $?$ & $?$ & + & $?$ & $?$ \\
\hline Xie ZL 2016 & $?$ & $?$ & $?$ & $?$ & $\oplus$ & $?$ & $\oplus$ \\
\hline Xu J 2016 & $?$ & $?$ & $?$ & $?$ & + & $?$ & + \\
\hline Xu L 2016 & ? & $?$ & $?$ & + & $\oplus$ & $?$ & $\oplus$ \\
\hline Zahra 2015 & ? & $?$ & $?$ & $?$ & $\oplus$ & $?$ & ? \\
\hline $\operatorname{lang}$ X 2015 & + & $?$ & ? & ? & $\oplus$ & $?$ & ? \\
\hline
\end{tabular}

(b)

Figure 2: $(\mathrm{a}, \mathrm{b})$ Risk of bias of included studies.

We performed sensitivity analysis by removing RCTs one by one; the results remained unchanged.

Subgroup analysis was performed based on the treatment duration. The results showed that there was no significant difference between $\mathrm{KT}$ and $\mathrm{CR}$ with treatment duration $\leq 4$ weeks $(\mathrm{MD}=5.03,95 \% \mathrm{CI}-1.80$ to $11.85, P=0.15 ; \mathrm{MD}=4.33$, $95 \% \mathrm{CI}-1.50$ to $10.15, P=0.15)$, while there was significant difference with treatment duration $>4$ weeks $(\mathrm{MD}=4.77,95 \% \mathrm{CI}$ 2.58 to $6.97, P<0.0001)$. Subgroup analysis was showed in Figure 4.

\subsubsection{Secondary Outcomes}

TUGT. 6 RCTs compared KT with CR [21, 23, 28, 33, 34, 38]. As shown in Figure 5, there was significant difference in
TUGT between KT and CR (MD=-4.62, 95\%CI -5.48 to -3.76 , $P<0.00001)$. We performed sensitivity analysis by removing RCTs one by one, the results remained unchanged.

FAC. 5 RCTs compared KT with CR $[24,26,29,35,36]$. As shown in Figure 6, there was significant difference in FAC between $\mathrm{KT}$ and $\mathrm{CR}(\mathrm{MD}=0.53,95 \% \mathrm{CI} 0.38$ to 0.68 , $P<0.00001)$. We performed sensitivity analysis by removing RCTs one by one, and the results remained unchanged. However, by removing Xie's study, the $I^{2}$ decreased from $93 \%$ to $40 \%$.

FMA-L. 9 RCTs compared KT with CR [22-24, 28, 29, 3436]. As shown in Figure 7, there was significant difference in FMA-L between KT and CR $(\mathrm{MD}=4.20,95 \% \mathrm{CI} 3.17$ to 5.24 , 


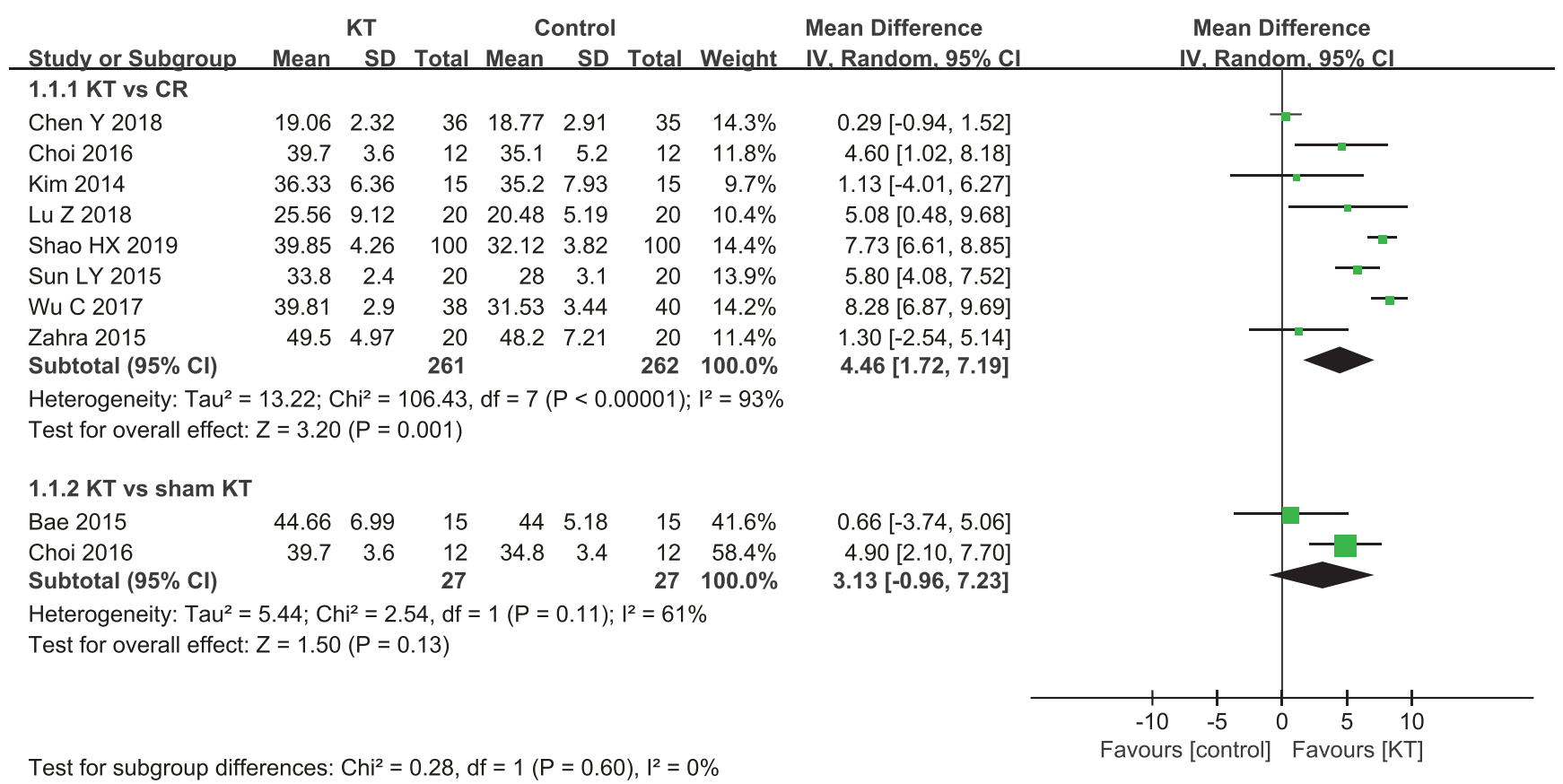

FIgURE 3: Meta-analysis results of KT for BBS.

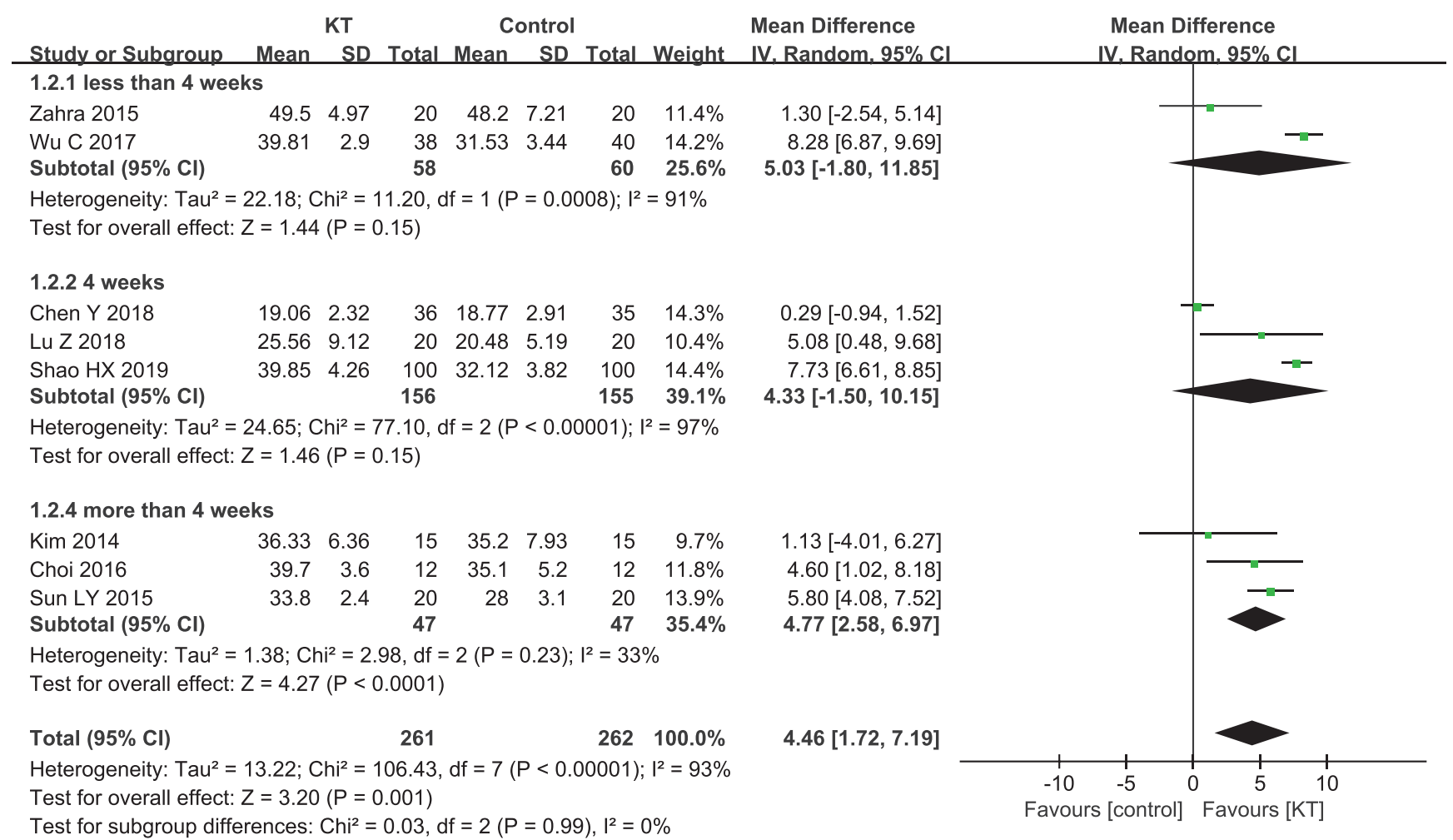

FIgURE 4: Subgroup analysis of KT for BBS.

$P<0.00001)$. We performed sensitivity analysis by removing RCTs one by one, and the results remained unchanged.

MAS. 5 RCTs compared KT with CR [25, 27, 28, 30, 33]. As shown in Figure 8, there was significant difference between $\mathrm{KT}$ and $\mathrm{CR}$ in MAS (MD $=-0.38,95 \% \mathrm{CI}-0.49$ to -0.27 ,
$P<0.00001)$. We performed sensitivity analysis by removing RCTs one by one, and the results remained unchanged.

Other Outcomes. As shown in Table 2, there was significant difference between $\mathrm{KT}$ and $\mathrm{CR}$ in 10mMVS $(\mathrm{MD}=0.23$, $95 \% \mathrm{CI} 0.06$ to $0.39, P=0.006)$, MMT ( $\mathrm{MD}=0.35,95 \% \mathrm{CI} 0.10$ 


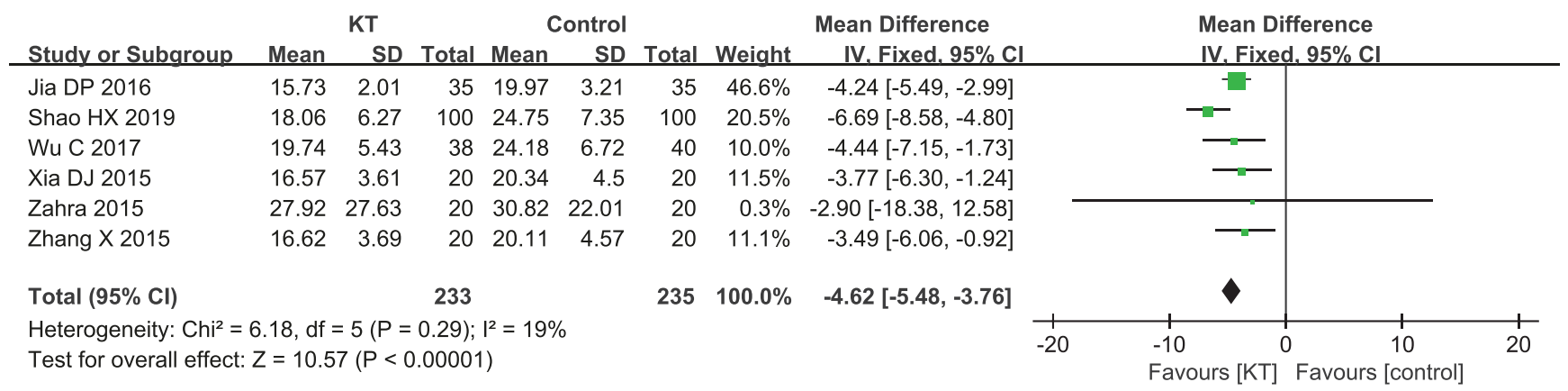

FIgURE 5: Meta-analysis results of KT for TUGT.

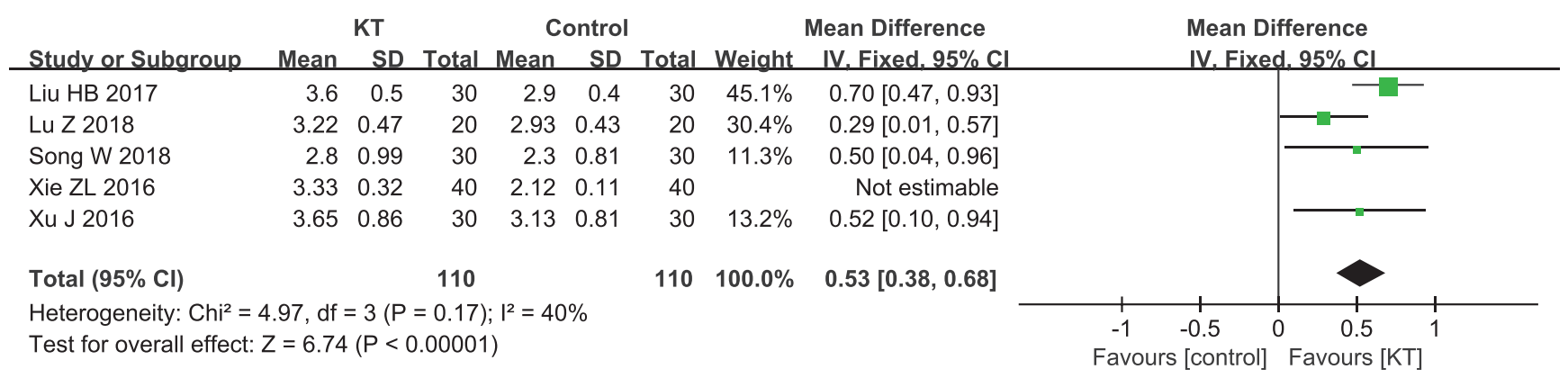

Figure 6: Meta-analysis results of KT for FAC.

\begin{tabular}{|c|c|c|c|c|c|c|c|c|c|c|c|c|}
\hline \multirow{2}{*}{$\begin{array}{l}\text { Study or Subgroup } \\
\text { Chen Y } 2018\end{array}$} & \multicolumn{2}{|c|}{${ }_{\text {Kean }}$ KT } & Total & & $\begin{array}{c}\text { ontrol } \\
\text { SD }\end{array}$ & Total & Weight & $\begin{array}{l}\text { Mean Difference } \\
\text { IV. Random, } 95 \% \mathrm{Cl}\end{array}$ & \multicolumn{3}{|c|}{$\begin{array}{c}\text { Mean Difference } \\
\text { IV. Random, } 95 \% \mathrm{Cl}\end{array}$} & \\
\hline & 21.14 & 4.3 & 36 & 19.34 & 3.05 & 35 & $10.5 \%$ & $1.80[0.07,3.53]$ & & & & \\
\hline Jia DP 2016 & 29.16 & 1.07 & 35 & 23.17 & 2.05 & 35 & $13.7 \%$ & $5.99[5.22,6.76]$ & & & - & \\
\hline Liu HB 2017 & 16.73 & 1.17 & 30 & 13.23 & 1.23 & 30 & $14.1 \%$ & $3.50[2.89,4.11]$ & & & - & \\
\hline Shao HX 2019 & 29.1 & 2.92 & 100 & 23.27 & 3.26 & 100 & $13.4 \%$ & $5.83[4.97,6.69]$ & & & - & \\
\hline Song W 2018 & 21.5 & 5.84 & 30 & 18.2 & 6 & 30 & $6.6 \%$ & $3.30[0.30,6.30]$ & & & & \\
\hline Xia DJ 2015 & 28.4 & 2.86 & 20 & 24.9 & 2.6 & 20 & $10.6 \%$ & $3.50[1.81,5.19]$ & & & & \\
\hline Xie ZL 2016 & 24.11 & 3.64 & 40 & 18.31 & 3.11 & 40 & $11.3 \%$ & $5.80[4.32,7.28]$ & & & & \\
\hline Xu J 2016 & 24.7 & 4.31 & 30 & 22.06 & 4.74 & 30 & $8.6 \%$ & $2.64[0.35,4.93]$ & & & & \\
\hline Zhang X 2015 & 28.09 & 2.42 & 20 & 24.13 & 2.41 & 20 & $11.3 \%$ & $3.96[2.46,5.46]$ & & & & \\
\hline Total $(95 \% \mathrm{Cl})$ & & & 341 & & & 340 & $100.0 \%$ & $4.20[3.17,5.24]$ & & & & \\
\hline $\begin{array}{l}\text { Heterogeneity: } \mathrm{Tau}^{2}= \\
\text { Test for overall effect: }\end{array}$ & $\begin{array}{l}1.89 ; \mathrm{Ch} \\
=7.95\end{array}$ & $\begin{array}{l}\mathrm{i}^{2}=51 \\
(\mathrm{P}<0\end{array}$ & $\begin{array}{l}.43, \mathrm{df} \\
.00001\end{array}$ & $=8(\mathrm{P}$ & $<0.000$ & $01) ; 1^{2}$ & $=84 \%$ & & -10 & $\begin{array}{ll}-5 & 0 \\
\text { urs [control] }\end{array}$ & $\begin{array}{c}5 \\
\text { Favours }[\mathrm{KT}]\end{array}$ & 10 \\
\hline
\end{tabular}

Figure 7: Meta-analysis results of KT for FMA-L.

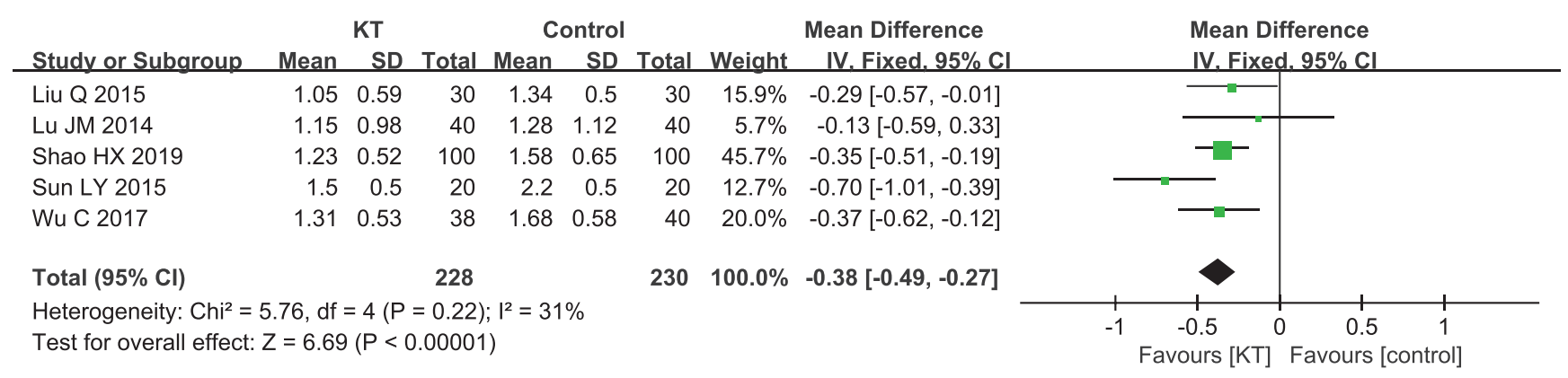

FIGURE 8: Meta-analysis results of KT for MAS. 
TABLE 2: Meta-analysis results of KT for other outcomes.

\begin{tabular}{lcccc}
\hline Outcome & No. of RCT & MD & $95 \%$ CI & $P$ value \\
\hline 10mMVS & 3 & 0.23 & $0.06,0.39$ & 0.006 \\
10mMWT & 3 & -3.68 & $-15.97,8.61$ & 0.56 \\
MMT & 2 & 0.35 & $0.10,0.59$ & 0.005 \\
AROM & 2 & 1.98 & $1.16,2.80$ & $<0.00001$ \\
Brunnstrom & 2 & 0.32 & $0.07,0.57$ & 0.01 \\
\hline
\end{tabular}

to $0.59, P=0.005)$, AROM ( $\mathrm{MD}=1.98,95 \% \mathrm{CI} 1.16$ to 2.80 , $P<0.00001)$ and Brunnstrom $(\mathrm{MD}=0.32,95 \% \mathrm{CI} 0.07$ to $0.57, P=0.01)$. However, there was no significant difference between KT and CR in 10mMWT (MD=-3.68, 95\%CI -15.97 to $8.61, P=0.56)$. $\mathrm{Xu} \mathrm{L}$ et al. [37] found that $\mathrm{KT}$ was more effective than CR on lower limb swelling in stroke patients. $\mathrm{Chen} \mathrm{Z}$ et al. [39] reported that KT can effectively decrease the angle of strephenopodia; it was safe with no adverse effects. Besides, Tan TC et al. [31] found that KT had a positive effect in improving gait and walking ability of hemiplegia patients. Tang Y et al. [32] found KT with physical therapy may be favorable in improving functional outcome of sit-tostand transfer in stroke patients and use on both sides is more effective.

\section{Discussion}

4.1. Summary of Findings. To our knowledge, this is the first meta-analysis of KT for balance ability after stroke. Results of this meta-analysis showed that $\mathrm{KT}$ was more effective than CR for balance ability, lower limb function, and walking function in poststroke patients. But no significant difference was found between $\mathrm{KT}$ and sham KT in BBS. Possible causes may be only two studies included. However, the results of BBS were not consistent regarding of the treatment duration. In the subgroup analysis, we found that, compared with $\mathrm{CR}$, KT was more effective with more than 4-week treatment duration. However, there was no significant difference between KT and CR with treatment duration $\leq 4$ weeks, which suggested that long-term KT may be more effective than short-term KT. Except 10mMWT, there was significant difference between $\mathrm{KT}$ and $\mathrm{CR}$ in TUGT, FMA, FAC, MAS, and other outcomes.

4.2. Implications for Further Studies. Balance impairment is one of the common daily functional problems in stroke patients, which is often associated with increasing risk of falls, poor walking function and low quality of life. KT is a safe and effective method, while the mechanism of KT is not clear at present, its therapeutic effect may be the stickiness and principles of kinematics and biomechanics, by sticking KT into different directions and using different tensions. Recent studies showed that KT had been used as an adjuvant rehabilitation therapy in the treatment of balance impairment after stroke. We retrieved 4 SRs of the clinical effects of KT [40-43], 1 SR was not only focus on stroke patients [40], and 3 of them focused on the motor function of lower extremity, which directly affected the balance function [41-43]. Compared with other outcomes, balance ability is the key for poststroke patients to return to family and society. 1 previous SR concluded that the current existing evidence was insufficient to support the use of KT over other modalities in clinical practice [40]. Due to these controversial conclusions, our SR focused on balance function and made a more comprehensive search in English and Chinese databases. 22 RCTs which met the inclusion criteria were included.

Results of Wu YH et al. [43] showed that 4-week KT was more effective than 6 weeks KT, which was opposite to our results. The possible reasons for the opposite result may be that $\mathrm{Wu}$ YH et al. combined BBS and TUGT together, which certainly contributed to heterogeneity. Our SR took BBS as primary outcome and TUGT as secondary outcome. Results of our SR indicated that KT was superior to CR in both BBS and TUGT.

4.3. Strengths and Limitations. This SR provides the latest evidence on KT for balance function after stroke based on the findings of relevant RCTs; the results of our meta-analysis showed that KT may be a beneficial complementary therapy in the balance rehabilitation process for stroke patients. The results of this study will provide evidence for the clinical application of KT. Different from current SRs, we conducted a comprehensive search and reporting in accordance with PRISMA (Appendix C). However, our study still has several limitations. First of all, the treatment duration of KT was inconsistent, from as short as an immediate treatment to 12 weeks, indicating a wide variation in designs. Second, the locations of KT were not standardized in lower limb, hip, thigh, and crus, which may also lead to discrepancy. Third, several outcomes included only 2 studies, which may lead to unreliable results.

Hence, more high methodological quality, larger sample size, and standard-designed trials are required to draw a definitive conclusion and provide a standard KT program.

\section{Conclusion}

Based on current evidence, this study demonstrated that KT was more effective than CR for balance ability, lower limb function and walking function in poststroke patients. Longer treatment duration may be associated with better effects. However, more well-conducted RCTs are required in the future. 


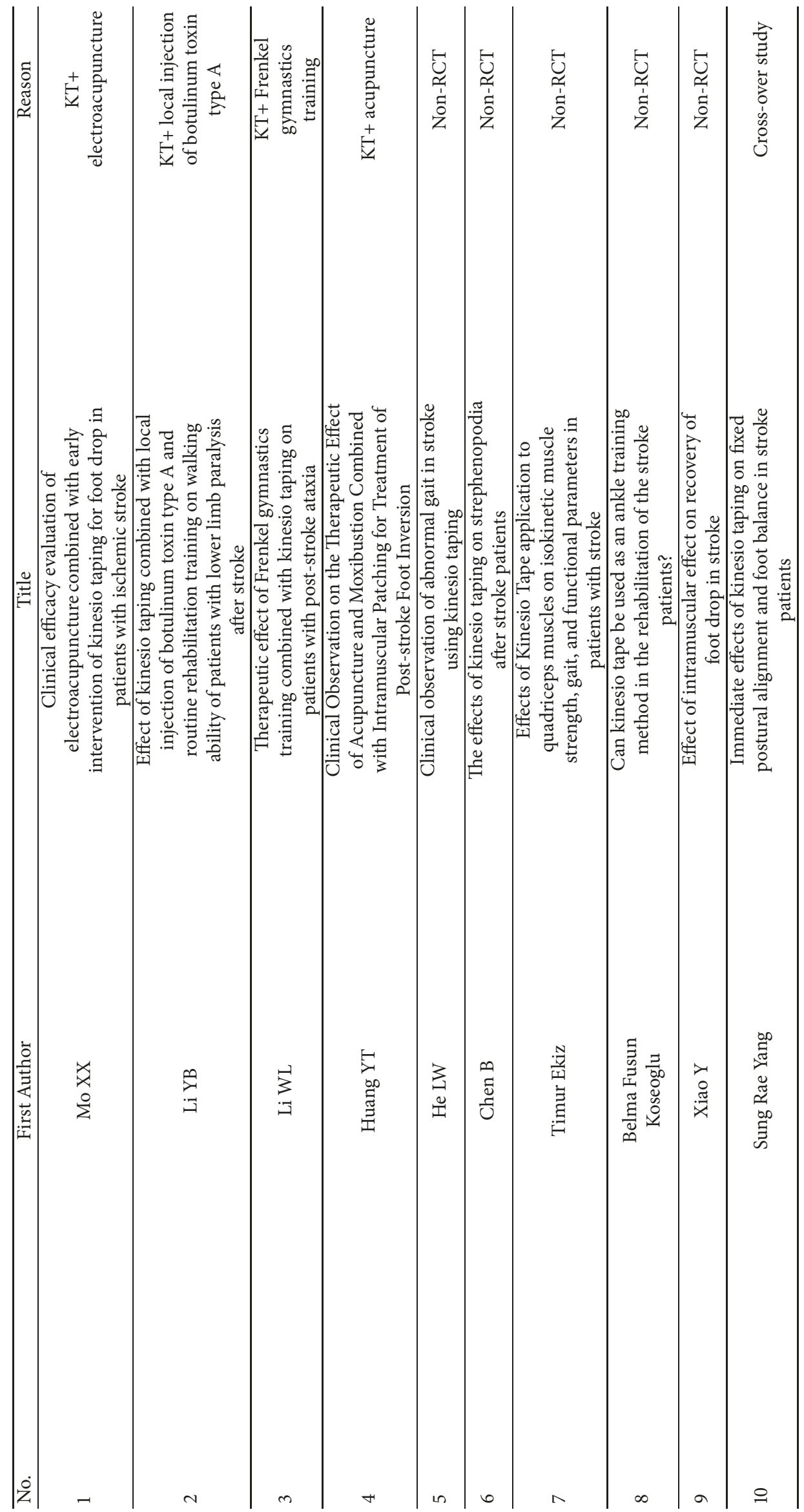




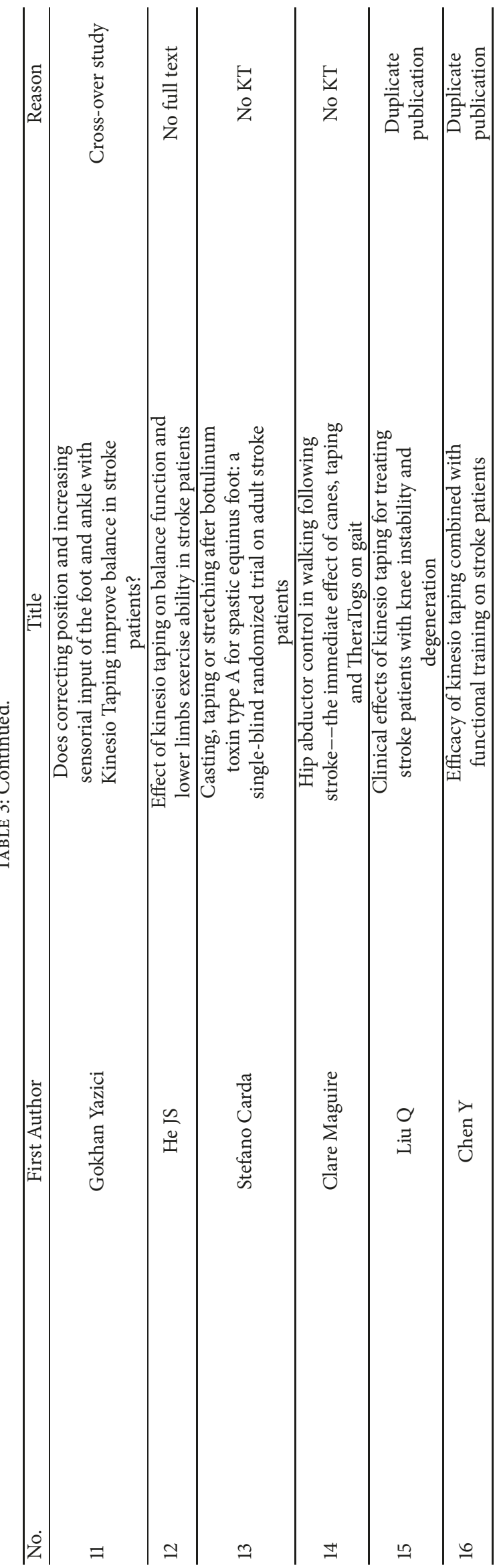




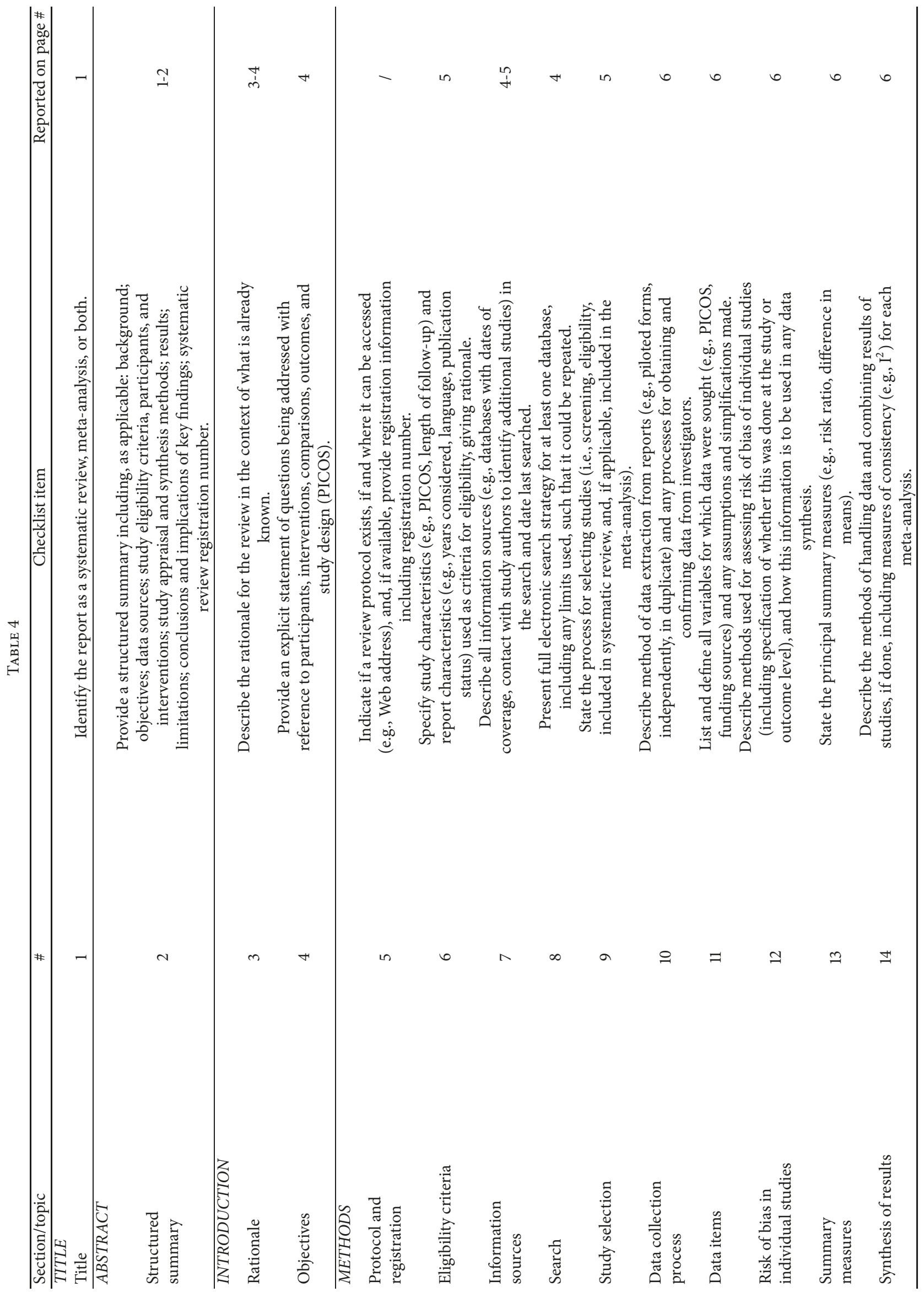




\section{Appendix}

\section{A. Search Strategy}

(1) Tape, Athletic OR Orthotic Tape OR Kinesio Tape OR Kinesio tape

(2) Strokes OR Cerebrovascular Accident OR CVA (Cerebrovascular Accident) OR Cerebrovascular Apoplexy OR Brain Vascular Accident OR Cerebrovascular Stroke OR Apoplexy OR Cerebral Stroke OR Acute Stroke OR Acute Cerebrovascular Accident

(3) (1) AND (2)

\section{B. Excluded Studies}

See Table 3.

\section{PRISMA Checklist}

See Table 4.

\section{Conflicts of Interest}

All authors declare that they have no conflicts of interest.

\section{Authors' Contributions}

Yijuan Hu, Dongling Zhong, and Qiwei Xiao contributed equally to the work as first authors. Rongjiang Jin and Juan $\mathrm{Li}$ contributed to study concept and design. Yijuan $\mathrm{Hu}$, Dongling Zhong, Qiwei Xiao, and Qiang Chen contributed to acquisition of data. Yijuan Hu, Dongling Zhong, Qiwei Xiao, and Juan Li contributed to drafting of the manuscript.

\section{Acknowledgments}

This work was financially funded by the National Natural Science Foundation of China (grant number 81873356) and the Sichuan Science and Technology Program (grant number 2019YFS0019).

\section{References}

[1] R. Sacco L, S. Kasner E, J. Broderick P et al., "An Updated Definition of Stroke for the 21st Century," Journal of Neurology and Neurorehabilitation, vol. 44, no. 7, pp. 2064-2089, 2064.

[2] "Asia Pacific Consensus Forum on Stroke Management Organizing Committees," Program, Advisory, and Local).

[3] S. Mendis, S. Davis, and B. Norrving, "Organizational update: the World Health Organization global status report on noncommunicable diseases 2014; one more landmark step in the combat against stroke and vascular disease," Stroke, vol. 46, no. 5, pp. e121-e122, 2015.

[4] X. Xu, E. Vestesson, L. Paley et al., "The economic burden of stroke care in England, Wales and Northern Ireland: Using a national stroke register to estimate and report patient-level health economic outcomes in stroke," European Stroke Journal, vol. 3, no. 1, pp. 82-91, 2017.
[5] H. Joo and D. Liang, "Economic burden of informal care attributable to stroke among those aged 65 years or older in China," International Journal of Stroke, vol. 12, no. 2, pp. 205207, 2017.

[6] S. F. Tyson, M. Hanley, J. Chillala, A. Selley, and R. C. Tallis, "Balance Disability After Stroke," Physical Therapy in Sport, vol. 86, no. 1, pp. 30-38, 2006.

[7] D. Hyndman, A. Ashburn, and E. Stack, "Fall events among people with stroke living in the community: circumstances of falls and characteristics of fallers," Archives of Physical Medicine and Rehabilitation, vol. 83, no. 2, pp. 165-170, 2002.

[8] E. L. Callaly, D. Ni Chroinin, N. Hannon et al., "Falls and fractures 2 years after acute stroke: the North Dublin Population Stroke Study," Age and Ageing, vol. 44, no. 5, pp. 882-886, 2015.

[9] P. Saari, E. Heikkinen, R. Sakari-Rantala, and T. Rantanen, "Fallrelated injuries among initially 75 - and 80 -year old people during a 10-year follow-up," Archives of Gerontology and Geriatrics, vol. 45, no. 2, pp. 207-215, 2007.

[10] C. I. Gryfe, A. Amies, and M. J. Ashley, "A longitudinal study of falls in an elderly population: I. Incidence and morbidity," Age and Ageing, vol. 6, no. 4, pp. 201-210, 1977.

[11] D. Wild, U. S. Nayak, and B. Isaacs, "Prognosis of falls in old people living at home," Journal of Epidemiology \& Community Health, vol. 35, no. 3, pp. 200-204, 1981.

[12] S. P. Baker and A. H. Harvey, "Fall Injuries in the Elderly," Clinics in Geriatric Medicine, vol. 1, no. 3, pp. 501-512, 1985.

[13] M. E. Tinetti, "Performance-orientated assessment of mobility problems in elderly patients," Journal of the American Geriatrics Society, vol. 34, no. 2, pp. 119-126, 1986.

[14] M. E. Tinetti, M. Speechley, and S. F. Ginter, "Risk factors for falls among elderly persons living in the community," The New England Journal of Medicine, vol. 319, no. 26, pp. 1701-1707, 1988.

[15] L. Nyberg and Y. Gustafson, "Patient falls in stroke rehabilitation: A challenge to rehabilitation strategies," Stroke, vol. 26, no. 5, pp. 838-842, 1995.

[16] E. Jaraczewska and C. Long, "Kinesio taping in stroke: Improving functional use of the upper extremity in hemiplegia," Topics in Stroke Rehabilitation, vol. 13, no. 3, pp. 31-42, 2006.

[17] S. R. Yang, S. Y. Heo, and H. J. Lee, "Immediate effects of kinesio taping on fixed postural alignment and foot balance in stroke patients," Journal of Physical Therapy Science, vol. 27, no. 11, pp. 3537-3540, 2015.

[18] W. I. Kim, Y. K. Choi, J. H. Lee, and Y. H. Park, "The effect of muscle facilitation using kinesio taping on walking and balance of stroke patients," Journal of Physical Therapy Science, vol. 26, no. 11, pp. 1831-1834, 2014.

[19] Y.-K. Choi, Y.-H. Park, and J.-H. Lee, "Effects of kinesio taping and McConnell taping on balance and walking speed of hemiplegia patients," Journal of Physical Therapy Science, vol. 28, no. 4, pp. 1166-1169, 2016.

[20] Y.-H. Bae, H. G. Kim, K. S. Min, and S. M. Lee, "Effects of Lower-Leg Kinesiology Taping on Balance Ability in Stroke Patients with Foot Drop," Evidence-Based Complementary and Alternative Medicine, vol. 2015, Article ID 125629, 5 pages, 2015.

[21] Z. Rojhani-Shirazi, S. Amirian, and N. Meftahi, "Effects of ankle kinesio taping on postural control in stroke patients," Journal of Stroke and Cerebrovascular Diseases, vol. 24, no. 11, pp. 25652571, 2015.

[22] Y. Chen, Effects of kinesio taping on lower limb function in patients with hemiplegia after early stroke, Southwestern Medical University, 2018. 
[23] D. P. Jia, L. Li, Z. Q. Wu et al., "Effect of intramuscular effect on the walking function of stroke patients with hemiplegia," China Medical Equipment, vol. 31, no. 80, p. S1, 2016.

[24] HB. Liu, P. Cai, and Y. Xiong, "Efficacy of kinesio taping in the improvement on walking ability of stroke patients with knee hyperextension," Chinese Journal of Trauma and Disability Medicine, vol. 25, no. 13, pp. 15-17, 2017.

[25] Q. Liu, Q. Tian, B. Yu et al., "Clinical observation of kinesio taping with physical therapy for improving functional outcome of stroke patients," Geriatrics \& Health Care, vol. 21, no. 5, pp. 284-286, 2015.

[26] Z. Lu, Y. Tang, and FD. Hu, "Effect of triggered functional electrical stimulation training combined with kinesio taping on walking ability of stroke patients with hemiplegia," Chinese Journal of Rehabilitation Medicine, vol. 33, no. 9, pp. 1102-1104, 2018.

[27] J. M. Lu, T. H. Gao, J. Jia et al., "Effect of kinesio taping on lower extremity function after stroke," Chinese Journal of Rehabilitation Medicine, vol. 29, no. 12, pp. 1165-1167, 2014.

[28] H. X. Shao, L. Ma, X. M. Liu et al., "Effect of Intramuscular Acombined with Rehabilitation Training on Lower Limb Motor Function, Walking Parameters and Quality of Life in Stroke Patients with Hemiplegia," Progress in Modern Biomedicine, vol. 19, no. 2, pp. 342-345, 2019.

[29] W. Song and DQ. Wang, "Observation of therapeutic effect of kinesio taping combined with rehabilitation training on stroke patients with foot drop," Chinese and Foreign Medical Research, vol. 16, no. 4, pp. 14-16, 2018.

[30] L. Y. Sun, X. M. Gao, N. Sun et al., "Clinical observation of different intramuscular patching methods in patients with hemiplegia after stroke," Medical Information, vol. 28, no. 8, pp. 104-105, 2015.

[31] TC. Tan, Ye. XM, and Yu. YM, "Effect of kinesio taping on gait in stroke patients with hemiplegia," Chinese Journal of Rehabilitation Medicine, vol. 31, no. 6, pp. 686-688, 2016.

[32] Y. Tang, J. Lin, and XA. Huang, "Kinesio taping on bilateral gluteus improves sit-to-stand transfer function in stroke patients," Zhejiang Medical Journal, vol. 39, no. 11, pp. 878-881, 2017.

[33] C. Wu, YL. Zhu, and Q. Liu, "Effect of kinesio taping-assisted lower limb training on lower extremity motor function in stroke patients with hemiplegia," Chinese Journal of Rehabilitation Medicine, vol. 32, no. 2, pp. 131-132, 2017.

[34] D. J. Xia, T. Peng, H. T. Wei et al., "Effect of kinesio taping of lower limbs on walking function in stroke patients with hemiplegia," Chinese Journal of Physical Medicine and Rehabilitation, vol. 37, no. 6, pp. 427-429, 2015 (Chinese).

[35] ZL. Xie, SW. Feng, and QR. Cao, "Application of kinesio taping in the prevention of knee extension in patients with hemiplegia after stroke [J]," Chinese Journal of Rehabilitation Medicine, vol. 31, no. 2, pp. 122-124, 2016.

[36] J. Xu, Hu. SH, and YF. Zhou, "Clinical observation of kinesio taping with physical therapy on the lower limb function and walking ability in hemiplegics stroke patients," Chinese Journal of Rehabilitation Medicine, vol. 31, no. 6, pp. 446-449, 2016.

[37] L. Xu, X. P. Li, and M. X. Gao, "Therapeutic effect of kinesio taping on lower extremity swelling in stroke patients," Journal of Huaihai Medicine, vol. 34, no. 6, pp. 683-684, 2016.

[38] X. Zhang, Du. LL, B. Zhao et al., "Therapeutic effect of kinesio taping on walking dysfunction in stroke patients with hemiplegia," Medical Information, vol. 28, no. 47, pp. 81-82, 2015.
[39] Z. Chen, XQ. Chen, and WJ. Che, "Clinical observation of kinesio taping on the treatment of post-stroke foot inversion," Journal of Bengbu Medical College, vol. 42, no. 12, pp. 1650-1652, 2017.

[40] D. Morris, D. Jones, H. Ryan, and C. G. Ryan, "The clinical effects of Kinesio Tex taping: a systematic review," Physiotherapy Theory and Practice, vol. 29, no. 4, pp. 259-270, 2013.

[41] N. Grampurohit, S. Pradhan, and D. Kartin, "Efficacy of adhesive taping as an adjunt to physical rehabilitation to influence outcomes post-stroke: a systematic review," Topics in Stroke Rehabilitation, vol. 22, no. 1, pp. 72-82, 2015.

[42] M. Wang, Z. Pei, B. Xiong, X. Meng, X. Chen, and W. Liao, "Use of Kinesio taping in lower-extremity rehabilitation of post-stroke patients: A systematic review and meta-analysis," Complementary Therapies in Clinical Practice, vol. 35, pp. 22-32, 2019.

[43] W. Yanhua, L. Zhuangmiao, and D. Xu, "Effects of kinesio taping at different intervention time on the motor function of lower extremity after stroke: a Meta-analysis," Chinese Journal of Tissue Engineering Research, vol. 22, no. 32, pp. 5241-5248, 2018. 


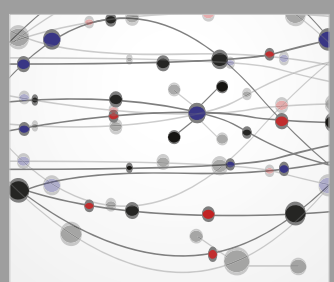

The Scientific World Journal
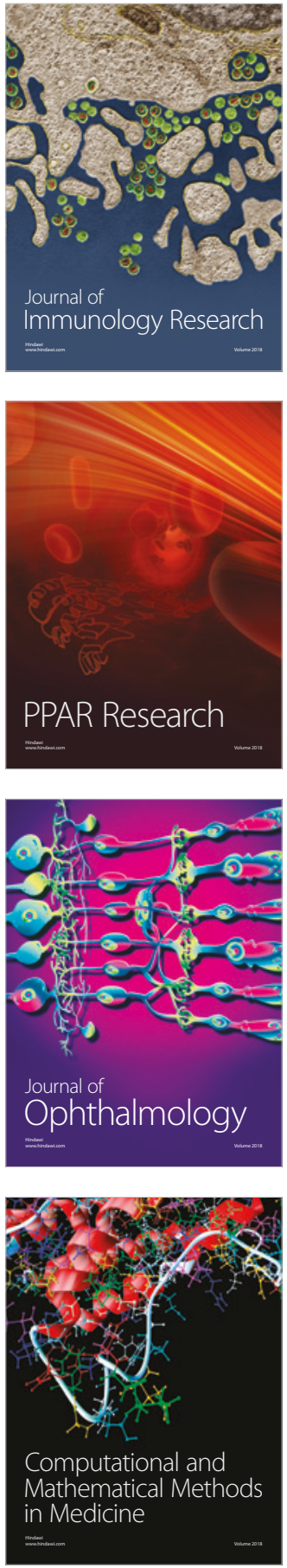

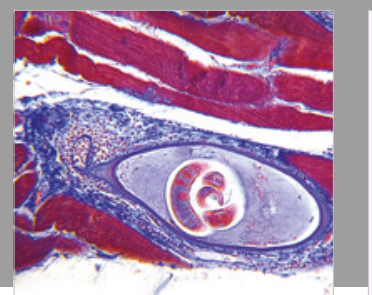

Gastroenterology Research and Practice

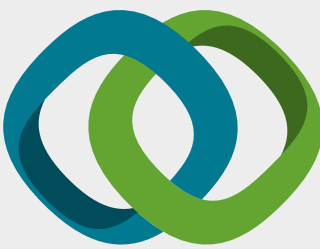

\section{Hindawi}

Submit your manuscripts at

www.hindawi.com
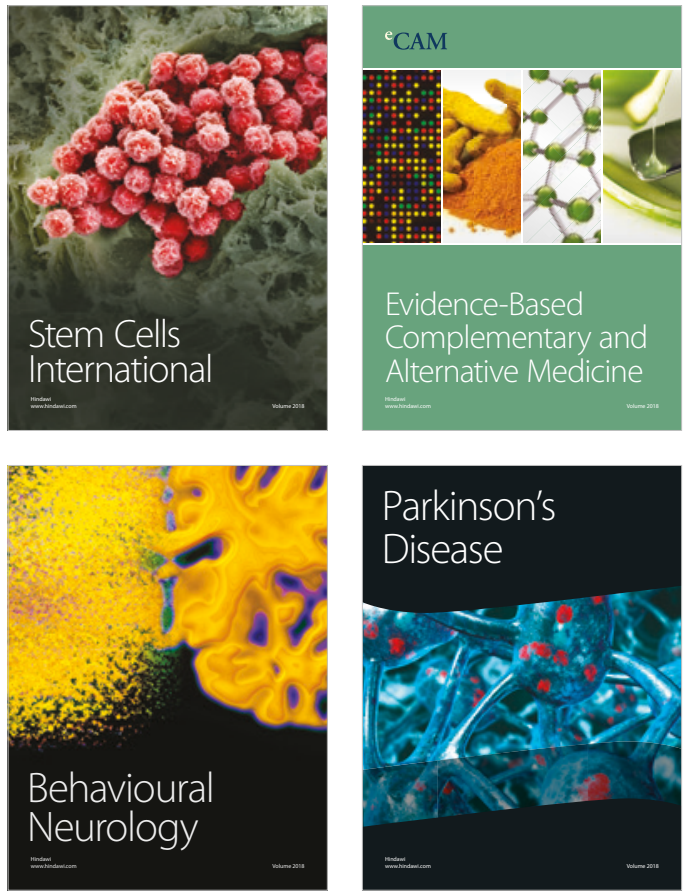

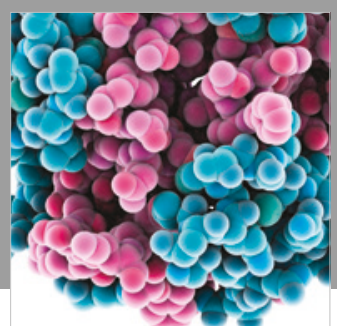

ournal of

Diabetes Research

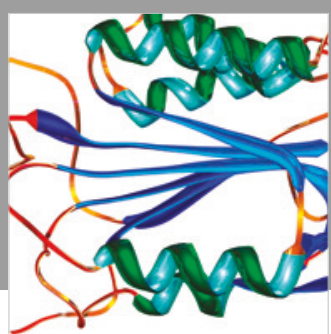

Disease Markers
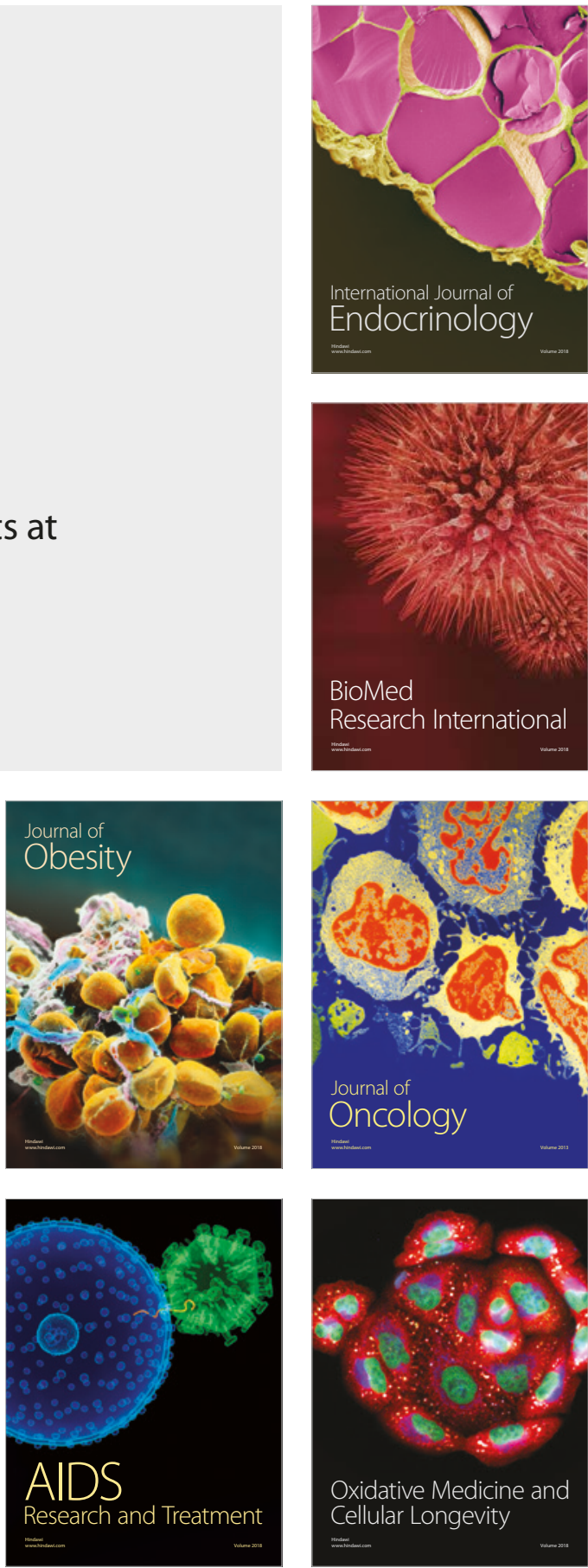